\title{
The influence of edge specific surface energy on the direction of hydrosilicate layers scrolling
}

\author{
A. A. Krasilin \\ Ioffe Institute, Politekhnicheskaya 26, St.-Petersburg, 194021, Russia \\ ikrasilin@mail.ioffe.ru
}

PACS 61.46.+w

DOI 10.17586/2220-8054-2021-12-5-623-629

\begin{abstract}
The present study reports on energy modeling of morphological features of hydrosilicate nanoscrolls with chrysotile structure. It considers a possibility of scrolling direction change driven by difference in specific surface energies on the hydrosilicate layer edges. Specific surface energy estimation together with energy modeling of the scrolling process reveal several directions, which are preferable in comparison to the [010] or [100] directions of scrolling. The results obtained may help to better understand correlation between morphology, structural features, and mechanical behavior of hydrosilicate nanoscrolls.
\end{abstract}

Keywords: inorganic nanotube, modeling, phyllosilicate, morphology.

Received: 11 August 2021

Revised: 25 September 2021

\section{Introduction}

Hydrosilicate layers like imogolite, halloysite, chrysotile, and their structural analogs may scroll due to size mismatch between octahedral and tetrahedral sheets and surface structure difference on the opposite sides of a layer [1]. The scrolling process yields nanoparticles of high specific surface area [2-5] having good mechanical properties [6-11] and oppositely charged inner and outer surfaces [5,12]. These remarkable features have promoted hydrosilicate nanoscrolls and nanotubes applications as catalysts [13-16], nanoreactors [17,18] and containers [19-21], polymer [22,23] and other functional materials [24] fillers.

Despite large variety of applications, formation mechanism, hydrosilicate nanoscrolls structure description, and understanding of related properties remain challenging. One particular problem relates to the form of cross-section (concentric rings, spiral or more complex forms [25]) and the direction of scrolling. A number of X-ray diffraction studies of chrysotile [26,27] and halloysite [28] came to the conclusion that the scrolling occurred along $b$ or seldom along $a$ axes (of monoclinic or triclinic unit cells). While this method gave integral information about nanotubes and nanoscrolls powders, electron diffraction patterns obtained on individual hydrosilicate and chalcogenide nanoscrolls [29-31] demonstrated deviations of scrolling direction from $a$ or $b$ axes. Concurrently, those particles were recognized as of cylindrical form. Strong deviations of scrolling direction or inequality of the scrolling driving force along the layer frequently leaded to formation of conical nanoscrolls [32-36]. An arbitrariness of scrolling direction may affect quality of hydrogen bonding between the adjacent layers [26], which, in turn, can play an important role in the mechanical behavior of hydrosilicate nanotubes and nanoscrolls $[8,10,11]$.

The present study has attempted to investigate the issue with scrolling direction of hydrosilicate layers from an energy modeling [37-42] perspective. Recent developments in the field of phenomenological modeling of scrolling [42] have revealed a crucial role for the specific surface energy $\sigma$ of the hydrosilicate layer edges in the choice between platy and scrolled particle shape. That study, however, operated with arbitrary range of edge $\sigma$ values. Here, edge $\sigma$ values have been estimated relative to certain $(h k 0)$ planes intersecting chrysotile unit cell. Thus, an assumption has been tested, that the direction of scrolling may deviate from directions of $a$ or $b$ axes because of energy preference of edges formed by $(h k 0)$ planes in comparison to the edges formed by (100) and (010) planes.

\section{Calculation concept}

\subsection{Energy model of scrolling}

An energy model of finite mass rectangular plate scrolling [41] was adopted for the calculation of energy effect value and preferable size parameters (number of layers and length) for cylindrical nanoscrolls with different $\sigma$ values on the edges. The model relied on three energy components, namely strain energy $E_{s}$, surface energy $\Sigma$, and adhesion energy $U_{a}$ :

$$
\Delta E_{/}^{@}=\frac{M}{m}\left[\left(E_{s}^{@}-E_{s}^{\prime}\right)+\left(\Sigma^{@}-\Sigma^{\prime}\right)-U_{a}^{@}\right]
$$


where $M$ is molar mass of the layer, $m$ is mass of the layer, @ sign denotes scrolled state, and / sign denotes flat state of the layer. The layer mass was held constant during the calculation and was determined as follows:

$$
m=L_{1} L_{2} h_{s} \rho=\text { const, }
$$

where $L_{1}$ is the length, along which the scrolling process occurred (equals to spiral length measured at $h_{s} / 2$ ), $L_{2}$ is the length of cylinder, $h_{s}$ is the layer thickness, and $\rho$ is density of the layer.

The strain energy of the scrolled state was determined within the continuous mechanics approach [43]:

$$
E_{s}^{@}=\frac{Y h_{s}^{3} L_{2}}{24(1-\mu)} \int_{0}^{2 \pi n}\left(\frac{1}{r_{i n}+f \varphi}-\frac{1}{r_{0}}\right)^{2} \sqrt{f^{2}+\left(r_{i n}+f \varphi\right)^{2}} d \varphi,
$$

where $Y$ is the Young's modulus, $\mu$ is the Poisson's ratio, $n$ is the number of layers (wraps), $r_{i n}$ is the inner radius of the nanoscroll, measured at $h_{s} / 2, r_{0}$ is the radius, at which size difference between octahedral and tetrahedral sheets is fully compensated by curvature (in the direction of scrolling), and $f=\left(h_{s}+t\right) / 2 \pi$ is the Archimedean spiral constant ( $t$ is the gap between adjacent layers). Square layer of side length $L_{r e f}$ was chosen as the reference flat state with the following strain energy:

$$
E_{s}^{\prime}=\frac{Y h_{s}^{3} L_{r e f}^{2}}{24(1-\mu) r_{0}^{2}}
$$

The surface energy was determined as sum of all surface areas multiplied by specific surface energy values:

$$
\Sigma^{@}=\sigma_{\text {out }} L_{1}^{\text {out }} L_{2}+\sigma_{\text {in }} L_{1}^{\text {in }} L_{2}+2 \sigma_{1} L_{1} h_{s}+2 \sigma_{2} L_{2} h_{s},
$$

where $\sigma_{\text {out }}, \sigma_{\text {in }}, \sigma_{1}, \sigma_{2}$ are specific surface energies of outer, inner, and two different edge surfaces, respectively, $L_{1}^{\text {out }}$ and $L_{1}^{i n}$ are lengths of the spiral beginning at $r=r_{i n} \pm h_{s} / 2$, respectively. The surface energy of the reference flat layer was written assuming $\sigma_{1}=\sigma_{2}=\sigma_{r e f}$ :

$$
\Sigma^{\prime}=L_{r e f}^{2}\left(\sigma_{\text {out }}+\sigma_{\text {in }}\right)+4 \sigma_{\text {ref }} L_{r e f} h_{s} .
$$

The adhesion energy at $n>1$ was estimated as follows:

$$
U_{a}^{@}=u_{a} L_{2} \int_{0}^{2 \pi(n-1)} \sqrt{f^{2}+\left(r_{i n}+\frac{h_{s}+t}{2}+f \varphi\right)^{2}} d \varphi
$$

where $u_{a}$ is the specific adhesion energy.

\subsection{Parametrization}

To determine energy effect of scrolling, the model must be parameterized. The mass of the layer (eq. (2)) was set to $10^{-15} \mathrm{~g}$, and the molar mass $277 \mathrm{~g} / \mathrm{mol}$ was calculated in accordance with the chemical formula $\mathrm{Mg}_{3} \mathrm{Si}_{2} \mathrm{O}_{5}(\mathrm{OH})_{4}$. The layer thickness $h_{s}=0.4 \mathrm{~nm}$ and the gap between adjacent layers $t=0.3 \mathrm{~nm}$ were chosen in accordance with electron microscopy study [10]. Density of the layer was set to $2.5 \mathrm{~g} / \mathrm{cm}^{3}$ [41]. The Young's modulus $Y=280 \mathrm{GPa}$ was calculated as an average of the data represented in theoretical paper [44], and the Poisson's ratio was set to 0.2 [45]. The stress-free radius $r_{0}=8.8 \mathrm{~nm}$ was taken from study [46]. Specific surface energies were chosen as follows: $\sigma_{\text {out }}=0.05 \mathrm{~J} / \mathrm{m}^{2}$ for (0001) surface of $\mathrm{Mg}(\mathrm{OH})_{2}$ brucite [47], $\sigma_{\text {in }}=1.51 \mathrm{~J} / \mathrm{m}^{2}$ for (111) surface of $\alpha-\mathrm{SiO} \mathrm{O}_{2}$ cristobalite [48]. An approach to calculation of specific surface energies at the edges of the layer is described in the subsection below. For the reference layer $\sigma_{r e f}=5.5 \mathrm{~J} / \mathrm{m}^{2}$ was chosen.

\subsection{Specific surface energy of the edges}

Specific surface energy calculation approach was based on the work of Shchipalov [48]. The $\sigma$ value was estimated as a quantity of broken chemical bonds per surface area. For an arbitrary $(h k l)$ plane specific surface energy was written as follows:

$$
\sigma_{h k l}=\frac{0.5}{N_{A} S_{h k l}} \sum_{i} E_{i} n_{i}^{b r},
$$

where $N_{A}$ is the Avogadro constant, $E_{i}$ is the energy of a single bond, $n_{i}^{b r}$ is the number of chemical bonds, intersected by the $(h k l)$ plane, and $S_{h k l}$ is the surface area of the $(h k l)$ plane, limited by periodicity condition. It was assumed that the edges of the layer are formed only by the $(h k 0)$ planes parallel to $c$ axis. Fig. 1 demonstrates an example of the $S_{h k 0}$ value determination. Whereas the relative position of chemical bonds and (010) or (110) planes reproduced from 
cell to cell, the (130) plane intersected the cell in 3 unique ways, so 3 cells must be considered. The final equation for the surface area was written as follows:

$$
S_{h k 0}=\sqrt{(h b)^{2}+(k a)^{2}} \cdot c \cdot \sin \beta,
$$

where $a, b, c$, and $\beta$ are lattice parameters. In the case shown in Fig. $1 a=0.5340 \mathrm{~nm}, b=0.9241 \mathrm{~nm}, c=1.4689 \mathrm{~nm}$, and $\beta=93.66^{\circ}(\sin \beta \cong 1)$.
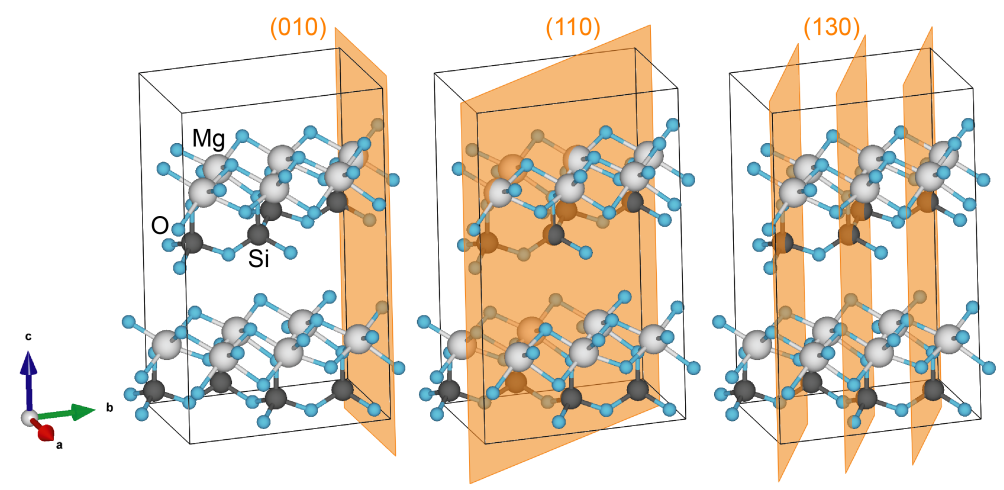

FIG. 1. Example of several lattice planes intersecting $\mathrm{Mg}_{3} \mathrm{Si}_{2} \mathrm{O}_{5}(\mathrm{OH})_{4}$ unit cell (hydrogen bonds between the layers are not shown). Structural data was adopted from [27] and visualized by VESTA software [50]

Only $\mathrm{Mg}-\mathrm{O}$ and $\mathrm{Si}-\mathrm{O}$ bonds were taken into account having bond energies $E_{\mathrm{Mg}-\mathrm{O}}=401 \pm 13 \mathrm{~kJ} / \mathrm{mol}$ [49] and $E_{\mathrm{Si}-\mathrm{O}}=444 \pm 18 \mathrm{~kJ} / \mathrm{mol}$ [48], respectively. Some of the $(h k 0)$ planes may intersect hydrogen bonds, however, their estimated energy $(5.2 \mathrm{~kJ} / \mathrm{mol}$ [42]) was almost two orders of magnitude less than those of $\mathrm{Mg}-\mathrm{O}$ and $\mathrm{Si}-\mathrm{O}$ bonds. In this regard, hydrogen bonds were omitted during the calculation. Edge surfaces were assumed to be oxide surfaces, without $\mathrm{OH}$-groups or water molecules adsorbed on them.

An angle between the (100) plane and $(h k 0)$ planes was calculated by using the following equations:

$$
\varphi_{100 \wedge h k 0}= \begin{cases}\frac{180}{\pi} \arccos \left(\frac{b}{\sqrt{(h b)^{2}+(k a)^{2}}}\right), & h= \pm 1, k \geq 1 \\ 90-\frac{180}{\pi} \arccos \left(\frac{b}{\sqrt{(h b)^{2}+(k a)^{2}}}\right), & h>1, k= \pm 1 .\end{cases}
$$

To model a rectangular plate, several $\sigma_{h k 0} / \sigma_{h^{\prime} k^{\prime} 0}$ pairs were chosen in accordance with a condition:

$$
\varphi_{100} \wedge h k 0+\varphi_{100 \wedge h^{\prime} k^{\prime} 0}=90 \pm 3^{\circ},
$$

where either $h^{\prime}$ or $k^{\prime}$ should be negative. Then $\sigma_{h k 0}$ substituted $\sigma_{1}$, and $\sigma_{h^{\prime} k^{\prime} 0}$ substituted $\sigma_{2}$ in the eq. (5).

\section{Results and discussion}

The choice of different $\sigma_{h k 0} / \sigma_{h^{\prime} k^{\prime} 0}$ pairs as specific surface energies of the edges $\left(\sigma_{1}\right.$ and $\sigma_{2}$, see eq. (5)) means that the rotation of the model rectangular plate undergoes in the $a b$ plane relative to the crystal structure. Consequently, direction of scrolling of the model plate would also change with the respect to the $a$ and $b$ axes. The results presented below were obtained within two important assumptions. The first one is that the $r_{0}$ value (eq. (3)) does not change with change in direction of scrolling, in other words, that the size difference between octahedral and tetrahedral sheets along any direction is the same. This assumption leads us to conclude that the hydrosilicate plate could be strained along any direction within the $a b$ plane. The second assumption is that the scrolling process occurs in only one direction, whereas strains along other directions either remain uncompensated or find another relaxation routes. One of these routes could be tetrahedron rotation yielding a tetrahedral sheet size change [37]. An alternative way for the crystal structure relaxation, strained in multiple directions, would be curving with formation of spherical particles. However, this case is rarely observed in practice: up to date, only allophane [51-53] demonstrates spherical morphology among hydrosilicates with the ability of curving. This feature could be related with the fact that allophane has the smallest $r_{0}$ value $(\sim 1 \mathrm{~nm})$ in comparison with other hydrosilicates [1]. 
Figure 2 shows the results of $\sigma_{h k 0}$ calculations. Both positive and negative $h k 0$ branches possessed several minima, which were relatively deep for the positive branch. The $h k 0$ values in the energy minima were 510,110 , 130, 1100 for the positive branch, and $4 \overline{1} 0, \overline{1} 20, \overline{1} 100$ for the negative branch. Both branches demonstrated similar trends at $\varphi_{100} \wedge h k 0$ being close to $0^{\circ}$ or $90^{\circ}$, whereas the trends were approximately opposite beyond that region. It is interesting to note, that $\sigma_{100}$ and $\sigma_{010}$ were among the highest values calculated. In the view of the necessity to choose $\sigma_{h k 0} / \sigma_{h^{\prime} k^{\prime}{ }_{0}}$ pairs in accordance with the eq. (11), this feature would make the particle with the edges formed by (100) and (010) planes comparatively less energetically preferable.

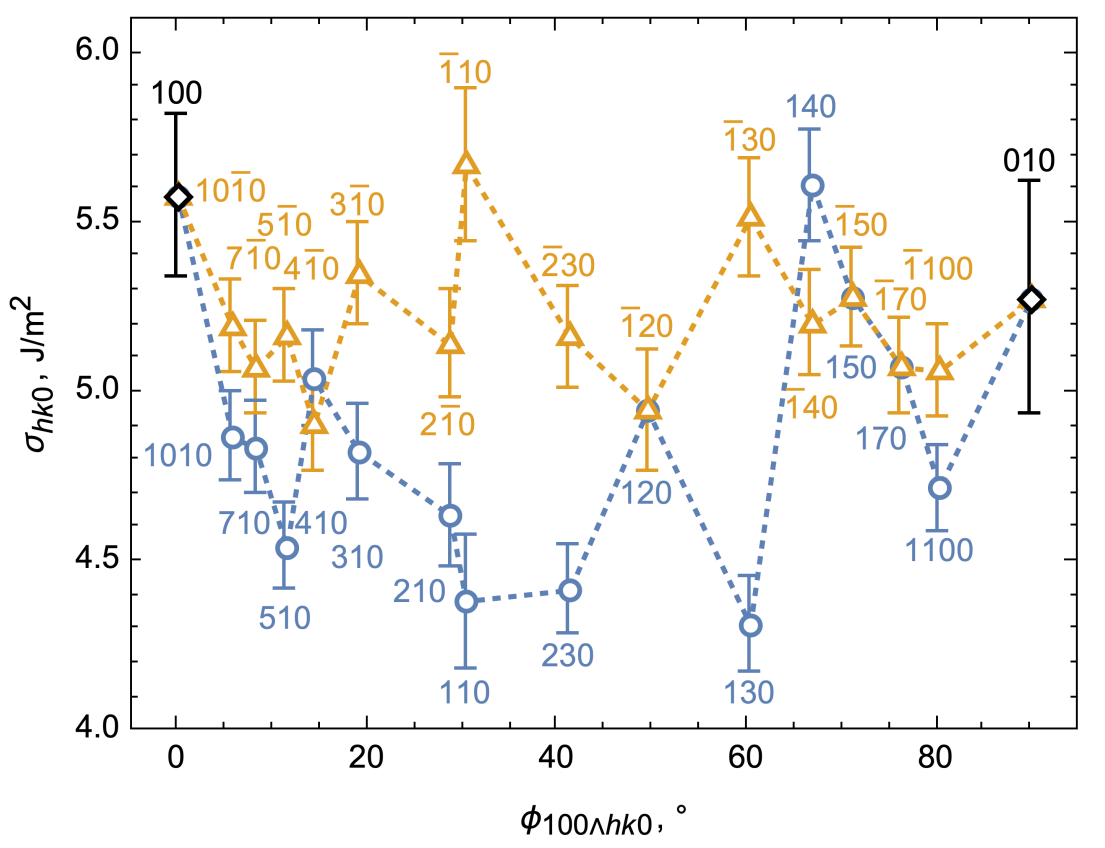

FIG. 2. Specific surface energies of ( $h k 0)$ planes vs. angle between the (100) and the $(h k 0)$ plane. The positive branch (both $h$ and $k$ are positive) is marked by blue circles, the negative branch (either $h$ or $k$ are negative) is marked by orange triangles. Dashed lines join points for guidance.

Figure 3(a) shows positions of energy minima of scrolled layers with the edges, formed by different pairs of $h k 0$ planes (110/130 pair as an example), perpendicular to each other (see eq. (11)). The angle $\varphi_{100 \wedge h k 0}$ is pointed for the plane with both $h$ and $k$ being positive (positive branch on Fig. 2). The relatively high value of energy effect was partially caused by the adhesion energy component (eq. (1)). The case of a multiwalled-nanoscroll is compared with that of a multilayered plate, for which the energy gap between them would be lower [42]. Depending on the $\sigma_{h k 0} / \sigma_{h^{\prime} k^{\prime} 0}$ pair, the energy effect could differ on approximately $400 \mathrm{~J} / \mathrm{mol}$, whereas the preferable spiral length $L_{1}$ almost did not change. Fig. 3(b) confirms miserable change of other energy preferable size parameters. The biggest change $(200 \mathrm{~nm})$ was revealed for cylinder length $L_{2}$. The results of calculation allow to assume that the hydrosilicate layers with different edge surfaces - within current approximation, scrolled in different directions could be morphologically indistinguishable, especially in the view of inner and outer diameters. On the other hand, variability of scrolling directions could broaden the nanoscroll length distribution. There is still a chance to determine scrolling direction by using the electron diffraction technique [30,54].

Figure 4 shows energy effect of scrolling (eq. (1)) of rectangular plates with edges formed by different $(h k 0)$ planes perpendicular to each other. The calculation revealed several energy minima with the deepest belonging to scrolling of $230 / \overline{1} 20$ plate. The general trend is almost symmetrical from both sides of this minimum. The 100/010 and 010/100 pairs, which are considered to be the most probable for scrolling of hydrosilicates like chrysotile and halloysite [28], were energetically unfavorable in the view of specific surface energy. At the same time, the 100/010 pair is slightly preferable than the $010 / 100$ pair. The nearest to $100 / 010$ pair energy minimum was formed by (510) and complementary (170) planes and was situated at $\varphi_{100 \wedge h k 0} \cong 10^{\circ}$. 



FIG. 3. (a) Change of energy minimum position for different $\sigma_{h k 0} / \sigma_{h^{\prime} k^{\prime} 0}$ pairs. Color denotes the angle between the (100) plane and the plane with positive $h$ and $k$. Complementary angle is determined by the eq. (11). (b) Energy preferable size parameters (inner diameter $d$, outer diameter $D$, and cylinder length $L_{2}$ ) of multiwalled nanoscrolls having different edge surfaces. Dashed lines join moving average values for guidance

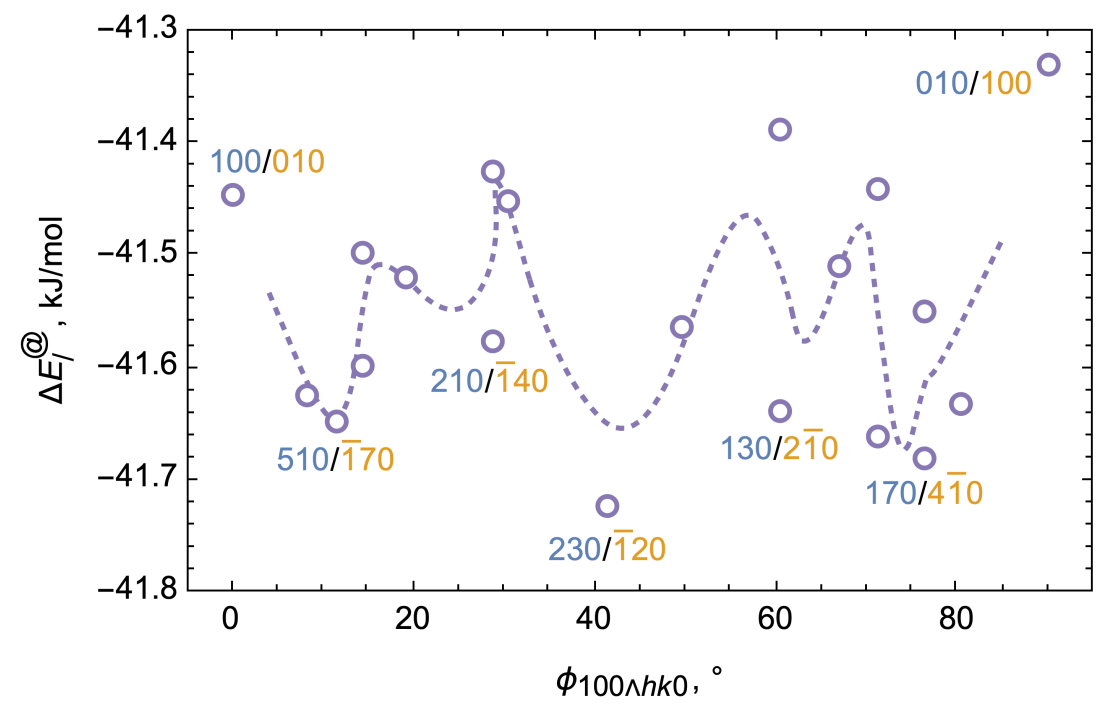

FIG. 4. Energy effect of scrolling for model rectangular plates with different edge surfaces. Local energy minima and 100/010 pairs are marked. Scrolling occurs along direction signed by orange text, whereas the spiral edge obtained is signed by blue text. Dashed line joins moving average values for guidance 


\section{Concluding remarks}

Specific surface energies of a family of $(h k 0)$ planes in $\mathrm{Mg}_{3} \mathrm{Si}_{2} \mathrm{O}_{5}(\mathrm{OH})_{4}$ chrysotile were estimated using a phenomenological approach and information on $\mathrm{Mg}-\mathrm{O}$ and $\mathrm{Si}-\mathrm{O}$ bond energies. It was shown that the specific surface energies of the main crystallographic planes (100) and (010) were among the highest among other values. The smallest values of $\sigma_{h k 0}$ were obtained for the (110), (230), and (130) planes.

The obtained data was used in calculation of energy effect of scrolling for a rectangular hydrosilicate model plate with the edge surfaces formed by $(h k 0)$ plane and complementary $(\bar{h} k 0)$ or $(h \bar{k} 0)$ plane. The calculations, carried out by the developed energy model, revealed several pairs of planes, at which energy effect of scrolling was maximal. All these pairs differed from 100/010 pair, although [010] direction is generally accepted as the main direction of hydrosilicate layer scrolling. The biggest energy effect of scrolling was revealed for 230/120 pair of planes, and the nearest to $100 / 010$ pair local energy minimum was revealed for 510/170 pair.

Preferable size parameters of nanoscroll models with different edge surfaces almost did not change being a possible obstacle for experimental distinguishing of nanoscrolls, which are cylindrically shaped but scrolled along different crystallographic directions, by electron microscopy. Energy modeling was carried out assuming direction of scrolling was always parallel to crystallographic direction of complementary plane. If this assumption is not correct because, for example, scrolling occurs along the crystallographic direction with maximal strains, then the formed scroll might still deviate from its cylindrical form. This deviation can be described as complication of the nanoscroll ends shape and formation of downward spiral on the side surface of the nanoscroll.

In the view of powder X-ray diffraction experiment, the direction of scrolling could be related with the crystallite size along certain direction. Crystallite size is expected to be the highest along that direction or in proximity to it.

\section{Acknowledgements}

The research was supported by the President of the Russian Federation grant MK-1962.2021.1.3. The author thanks Dr. A. V. Ankudinov, Dr. A. A. Levin, and Prof. V. V. Gusarov for fruitful discussions.

\section{References}

[1] Krasilin A.A., Khrapova E.K., Maslennikova T.P. Cation doping approach for nanotubular hydrosilicates curvature control and related applications. Crystals, 2020, $10(8), 654$.

[2] Shafia E., Esposito S., et al. A1/Fe isomorphic substitution versus Fe2O3 clusters formation in Fe-doped aluminosilicate nanotubes (imogolite). J. Nanopart. Res., 2015, 17 (8), 336.

[3] Zsirka B., Táborosi A., et al. Surface characterization of mechanochemically modified exfoliated halloysite nanoscrolls. Langmuir, 2017, 33 (14), P. 3534-3547.

[4] Lesci I.G., Balducci G., et al. Surface features and thermal stability of mesoporous Fe doped geoinspired synthetic chrysotile nanotubes. Micropor. Mesopor. Mat., 2014, 197, P. 8-16.

[5] Krasilin A.A., Danilovich D.P., et al. Crystal violet adsorption by oppositely twisted heat-treated halloysite and pecoraite nanoscrolls. Appl. Clay Sci., 2019, 173, P. 1-11.

[6] Guimarães L., Enyashin A.N., et al. Imogolite nanotubes: stability, electronic, and mechanical properties. ACS Nano, 2007,1 (4), P. 362-368.

[7] Guimarães L., Enyashin A.N., Seifert G., Duarte H.A. Structural, electronic, and mechanical properties of single-walled halloysite nanotube models. J. Phys. Chem. C, 2010, 114 (26), P. 11358-11363.

[8] Lecouvet B., Horion J., et al. Elastic modulus of halloysite nanotubes. Nanotechnology, 2013, 24 (10), 105704.

[9] Kumzerov Y.A., Parfen'eva L.S., et al. Thermal and acoustic properties of chrysotile asbestos. Phys. Solid State, 2005,47 (2), P. $370-373$.

[10] Piperno S., Kaplan-Ashiri I., et al. Characterization of geoinspired and synthetic chrysotile nanotubes by atomic force microscopy and transmission electron microscopy. Adv. Funct. Mater., 2007, 17 (16), P. 3332-3338.

[11] Khalisov M.M., Lebedev V.A., et al. Young's modulus of phyllosilicate nanoscrolls measured by the AFM and by the in-situ TEM indentation. Nanosystems: Phys. Chem. Math., 2021, 12 (1), P. 118-127.

[12] Lisuzzo L., Cavallaro G., et al. Colloidal stability of halloysite clay nanotubes. Ceram. Int., 2019, 45 (2), P. 2858-2865.

[13] Bonelli B., Bottero I., et al. IR spectroscopic and catalytic characterization of the acidity of imogolite-based systems. J. Catal., 2009, 264 (1), P. $15-30$.

[14] Mahajan A., Gupta P. Halloysite nanotubes based heterogeneous solid acid catalysts. New J. Chem., 2020, 44 (30), P. 12897-12908.

[15] Bian Z., Kawi S. Preparation, characterization and catalytic application of phyllosilicate: A review. Catal. Today, 2020,339 , P. 3-23.

[16] Khrapova E.K., Ugolkov V.L., et al. Thermal behavior of Mg-Ni-phyllosilicate nanoscrolls and performance of the resulting composites in hexene-1 and acetone hydrogenation. ChemNanoMat, 2021, 7 (3), P. 257-269.

[17] Monet G., Paineau E., et al. Solid wetting-layers in inorganic nano-reactors: the water in imogolite nanotube case. Nanoscale Adv., 2020, 2 (5), P. 1869-1877.

[18] Pignié M.-C., Shcherbakov V., et al. Confined water radiolysis in aluminosilicate nanotubes: the importance of charge separation effects. Nanoscale, 2021, 13 (5), P. 3092-3105.

[19] Lvov Y., Wang W., Zhang L., Fakhrullin R. Halloysite clay nanotubes for loading and sustained release of functional compounds. Adv. Mater., 2016, 28 (6), P. 1227-1250.

[20] Cavallaro G., Milioto S., Lazzara G. Halloysite nanotubes: interfacial properties and applications in cultural heritage. Langmuir, 2020, 36 (14), P. 3677-3689. 
[21] Maslennikova T.P., Korytkova E.N. Aqueous solutions of cesium salts and cesium hydroxide in hydrosilicate nanotubes of the $\mathrm{Mg}_{3} \mathrm{Si}_{2} \mathrm{O}_{5}(\mathrm{OH})_{4}$ composition. Glass Phys. Chem., 2010, 36 (3), P. 345-350.

[22] Kononova S.V., Korytkova E.N., et al. Polymer-inorganic nanocomposites based on aromatic polyamidoimides effective in the processes of liquids separation. Russ. J. Gen. Chem., 2010, 80 (6), P. 1136-1142.

[23] Lvov Y., Abdullayev E. Functional polymer-clay nanotube composites with sustained release of chemical agents. Prog. Polym. Sci., 2013, 38 (10-11), P. 1690-1719.

[24] Khrapova E.K., Ezhov I.S., et al. Nanotubular nickel hydrosilicate and its thermal annealing products as anode materials for lithium ion batteries. Inorg. Mater., 2020, 56 (12), P. 1248-1257,

[25] Yada K. Study of microstructure of chrysotile asbestos by high-resolution electron microscopy. Acta Crystallogr. A, 1971, 27 (6), P. 659-664.

[26] Wicks F.J., Whittaker E.J.W. A reappraisal of the structures of the serpentine minerals. Can. Mineral., 1975, 13 (3), P. $227-243$.

[27] Falini G., Foresti E., et al. Tubular-shaped stoichiometric chrysotile nanocrystals. Chem. Eur. J., 2004, 10 (12), P. $3043-3049$.

[28] Drits V.A., Sakharov B.A., Hillier S. Phase and structural features of tubular halloysite (7 Å). Clay Miner., 2018, 53 (4), P. 691-720.

[29] Niu J., Qiang Y., et al. Morphology and orientation of curling of kaolinite layer in hydrate. Appl. Clay Sci., 2014, 101, P. $215-222$.

[30] Khalitov Z., Khadiev A., Pashin D. Electron diffraction patterns from scroll nanotubes: interpretation peculiarities. J. Appl. Crystallogr., 2015, 48 (1), P. 29-36.

[31] Makó É., Dódony I., et al. Nanoscale structural and morphological features of kaolinite nanoscrolls. Appl. Clay Sci., 2020, 198, 105800.

[32] Korytkova E.N., Maslov A.V., et al. Formation of $\mathrm{Mg}_{3} \mathrm{Si}_{2} \mathrm{O}_{5}(\mathrm{OH})_{4}$ nanotubes under hydrothermal conditions. Glass Phys. Chem., 2004, 30 (1), P. 51-55.

[33] Krasilin A.A., Suprun A.M., Nevedomsky V.N., Gusarov V.V. Formation of conical $(\mathrm{Mg}, \mathrm{Ni})_{3} \mathrm{Si}_{2} \mathrm{O}_{5}(\mathrm{OH})_{4}$ nanoscrolls. Dokl. Phys. Chem., 2015,460 (2), P. 42-44.

[34] Krasilin A.A., Gusarov V.V. Redistribution of Mg and Ni cations in crystal lattice of conical nanotube with chrysotile structure. Nanosystems: Phys. Chem. Math., 2017, 8 (5), P. 620-627.

[35] Bloise A., Barrese E., Apollaro C. Hydrothermal alteration of Ti-doped forsterite to chrysotile and characterization of the resulting chrysotile fibers. Neues Jb. Miner. Abh., 2009, 185 (3), P. 297-304.

[36] Bloise A., Belluso E., et al. Hydrothermal alteration of glass to chrysotile. J. Am. Ceram. Soc., 2012 , 95 (10), P. $3050-3055$.

[37] Singh B. Why does halloysite roll? - A new model. Clay. Clay Miner., 1996, 44 (2), P. 191-196.

[38] Perbost R., Amouric M., Olives J. Influence of cation size on the curvature of serpentine minerals: HRTEM-AEM study and elastic theory. Clay. Clay Miner, 2003, 51 (4), P. 430-438.

[39] Chivilikhin S.A., Popov I.Y., Gusarov V.V. Dynamics of nanotube twisting in a viscous fluid. Dokl. Phys., 2007,52 (1), P. 60-62.

[40] Thill A., Guiose B., et al. How the diameter and structure of $(\mathrm{OH})_{3} \mathrm{Al}_{2} \mathrm{O}_{3} \mathrm{Si}_{x} \mathrm{Ge}_{1-x} \mathrm{OH}$ imogolite nanotubes are controlled by an adhesion versus curvature competition. J. Phys. Chem. C, 2012, 116 (51), P. 26841-26849.

[41] Krasilin A.A., Nevedomsky V.N., Gusarov V.V. Comparative energy modeling of multiwalled $\mathrm{Mg}_{3} \mathrm{Si}_{2} \mathrm{O}_{5}\left(\mathrm{OH}_{4}\right.$ and Ni $\mathrm{Si}_{2} \mathrm{O}_{5}\left(\mathrm{OH}_{4}\right)_{4}$ nanoscroll growth. J. Phys. Chem. C, 2017, 121 (22), P. 12495-12502.

[42] Krasilin A.A. Energy modeling of competition between tubular and platy morphologies of chrysotile and halloysite layers. Clay. Clay Miner. 2020,68 (5), P. 436-445.

[43] Landau L.D., Pitaevskii L.P., Kosevich A.M., Lifshitz E.M. Theory of Elasticity, Third Edition: Volume 7 (Course of Theoretical Physics), Butterworth-Heinemann, Oxford, 1986, 195 p.

[44] Lourenço M.P., de Oliveira C., et al. Structural, electronic, and mechanical properties of single-walled chrysotile nanotube models. J. Phys. Chem. C, 2012, 116 (17), P. 9405-9411.

[45] Cressey B.A., Whittaker E.J.W. Five-fold symmetry in chrysotile asbestos revealed by transmission electron microscopy. Mineral. Mag., 1993, 57 (389), P. 729-732.

[46] Demichelis R., De La Pierre M., et al. Serpentine polymorphism: a quantitative insight from first-principles calculations. CrystEngComm, 2016, 18 (23), P. 4412-4419.

[47] Churakov S.V., Iannuzzi M., Parrinello M. Ab initio study of dehydroxylation?carbonation reaction on brucite surface. J. Phys. Chem. B, 2004, 108 (31), P. 11567-11574.

[48] Shchipalov Y.K. Surface energy of crystalline and vitreous silica. Glass Ceram., 2000, 57 (11-12), P. $374-377$.

[49] Cotton D.H., Jenkins D.R. Bond-dissociation energy of gaseous magnesium oxide. T. Faraday Soc., 1969, 65, P. 376-379.

[50] Momma K., Izumi F. VESTA 3 for three-dimensional visualization of crystal, volumetric and morphology data. J. Appl. Crystallogr., 2011, 44 (6), P. 1272-1276.

[51] Parfitt R.L. Allophane and imogolite: role in soil biogeochemical processes. Clay Miner., 2009, 44 (1), P. $135-155$.

[52] Du P., Yuan P., et al. Insights into the formation mechanism of imogolite from a full-range observation of its sol-gel growth. Appl. Clay Sci., 2017, 150, P. 115-124.

[53] Thill A., Picot P., Belloni L. A mechanism for the sphere/tube shape transition of nanoparticles with an imogolite local structure (imogolite and allophane). Appl. Clay Sci., 2017, 141, P. 308-315.

[54] Khadiev A., Khalitov Z. Quantitative theory of diffraction by cylindrical scroll nanotubes. Acta Crystallogr. A, 2018,74 (3), P. $233-244$. 